\title{
Opinion Formation on a Deterministic Pseudo-fractal Network
}

\author{
M.C. González ${ }^{1}$, A.O. Sousa ${ }^{2}$, H.J. Herrmann ${ }^{3}$ \\ Institute for Computer Applications 1 (ICA1), University of Stuttgart, \\ Pfaffenwaldring 27, 70569 Stuttgart, Germany.
}

\begin{abstract}
The Sznajd model of socio-physics, that only a group of people sharing the same opinion can convince their neighbors, is applied to a scale-free random network modeled by a deterministic graph. We also study a model for elections based on the Sznajd model and the exponent obtained for the distribution of votes during the transient agrees with those obtained for real elections in Brazil and India. Our results are compared to those obtained using a Barabási-Albert scale-free network.

Keywords: Social systems, Deterministic graphs, Small-world networks, Scale-free networks, Elections
\end{abstract}

\section{Introduction}

Growing interest has been focused on the statistical physics of complex networks. They describe a wide range of systems in nature and society, modeling diverse systems as the Internet, the World Wide Web, the net of human sexual contacts, a network of chemicals linked by chemical reactions, and social and biological networks [1, 2, 3, 4, 5, 6. Such networks exhibit a rich set of scaling properties. A number of them are scale-free, that is, the probability that a randomly selected node has exactly $k$ links decays as a power law, following $P(k) \sim k^{-\gamma}$, where $\gamma$ is the degree exponent. Consequently they present resilience against random breakdowns, an effect known as robustness of the network. The shortest-path length between their sites grows slowly (i.e. logarithmically) with the size of the network. That is, compared to the large sizes of networks, the distance between their sites are short - a feature known as the "small world" effect.

The majority of networks used to generate a scale-free topology are stochastic, i.e. the nodes appear to be randomly connected to each other. These scalefree random networks have naturally a continuous degree distribution. But recently it has been shown that discrete degree distributions of some deterministic graphs asymptotically also exhibit a power law decay [3]. Furthermore, scalefree random networks are excellently modeled by such deterministic graphs 4 . However the comparison between the behavior of stochastic and deterministic networks in the simulation of a particular model still remains open.

The involvement of physics in research on spreading of opinion has been increasing [7, 8. Of particular interest here is the Sznajd model 9], which is one

\footnotetext{
${ }^{1}$ marta@ica1.uni-stuttgart.de

${ }^{2}$ sousa@ica1.uni-stuttgart.de

3hans@ica1.uni-stuttgart.de
} 
of several recent consensus-finding models [8] 10, and in which each randomly selected pair of nearest neighbors convinces all its neighbors of their opinion, only if the pair shares the same opinion ("Together we stand"); otherwise, the neighbor opinions are not affected. One time step means that on average every lattice node is selected once as the first member of the pair. It differs from other consensus models by dealing only with communication between neighbors, and the information flows outward, as in rumor spreading. In contrast, in most other models the information flows inward. Initially, two opinions $(+1$ and -1$)$ are randomly distributed with probability $p$ and $1-p$ respectively over all the nodes of the lattice. The basic Sznajd model with random sequential updating always leads to a consensus (all sites have the same opinion and the whole system reaches a fixed point after a certain simulation time). A phase transition is often observed as a function of the initial concentration of opinion $p$. A generalization to many different opinions (instead of only \pm 1 ) simulated on a Barabási-Albert network [1] reproduced quite well the results of the complex elections of city councillors in the state of Minas Gerais in Brazil in 1998 [12.

In the next section we present the deterministic scale-free network. Then we simulate using the Sznajd model an election process on such a network. We compare our results with the same simulations carried out on a stochastic scale-free network (the Barabási-Albert network) and with states election's from Brazil and India.



$t=0$

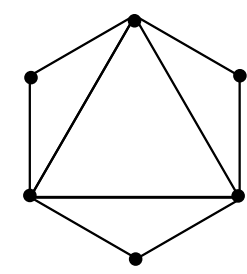

$t=1$

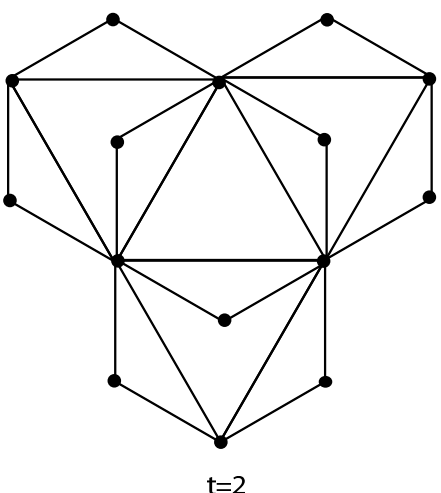

$t=2$

Figure 1: The three first generations of the scale-free pseudo-fractal graph. At each iteration step $t$, every edge generates an additional vertex, which is attached to the two vertices of this edge.

\section{Models and Results}

The deterministic scale-free graph used in this work grows as follows: At each time step, every edge generates an additional vertex, which is attached to both end vertices of this edge. Initially, at $t=0$, we have a triangle of edges connecting three vertices, at $t=1$, the graph consists of 6 vertices connected by 9 
edges, and so on (see Fig प). The total number of vertices at iteration $t$ is

$$
N_{t}=\frac{3\left(3^{t}+1\right)}{2}
$$

This simple rule produces a complex growing network. Such a graph is called a pseudo-fractal. It has a discrete degree distribution. To relate the exponent of this discrete degree distribution to the standard $\gamma$ exponent of a continuous degree distribution for random scale-free networks, we use a cumulative distribution $P_{\text {cum }}(k)$, which follows

$$
P_{\text {cum }}(k) \sim k^{1-\gamma}
$$

and it is the probability that a node of the network hat at least $k$ neighbors. It decreases as a power of $k$ with exponent $\gamma=1+\ln 3 / \ln 2$. In a similar way the average clustering coefficient 4], which is the probability of existence of a link between two nodes when they are both neighbors of a same node, can be calculated for the infinite graph, $\bar{C}=4 / 5$. One obtains a shortest-path-length distribution which tends to a Gaussian of width $\sim \sqrt{\ln N}$ centered at $\bar{l} \sim \ln N$ for large networks 4 . These properties concerning the degree distribution, the clustering coefficient and the mean length, are also present in a wide range of stochastic scale-free networks reported in the literature. They make our simple deterministic networks suitable to examine applications very often found on stochastic networks. In the next section we apply the Sznajd model on our scale-free pseudo-fractal.

\subsection{Monte Carlo simulations of the Sznajd model}

We let the fractal of Fig 1 grow and at each step assign sites with random opinion \pm 1 . At every step $t>0$, we have the following process:

1. The network grows, i.e., $3^{t}$ new sites are added.

2. A random opinion $( \pm 1)$ is set to each new node of the network, with probability $p(1-p)$ for opinion $+1(-1)$.

3. $N_{s}$ Sznajd runs are performed. For each run, $3^{t}$ sites chosen randomly are analyzed and updated, i.e., one visits for the Sznajd model a number of sites equal to the number of sites added at that step to the network.

Three variations of the Sznajd model on the pseudo-fractal network have been investigated:

- 1 site convincing: For each site $i$ chosen, we change the opinion of all its neighbors to the site's opinion.

- 2 sites convincing: For each site $i$ chosen, we select randomly one of its neighbors. If this selected neighbor has the same opinion of the site $i$, then all their neighbors follow the pair's opinion. Otherwise, nothing is done. 


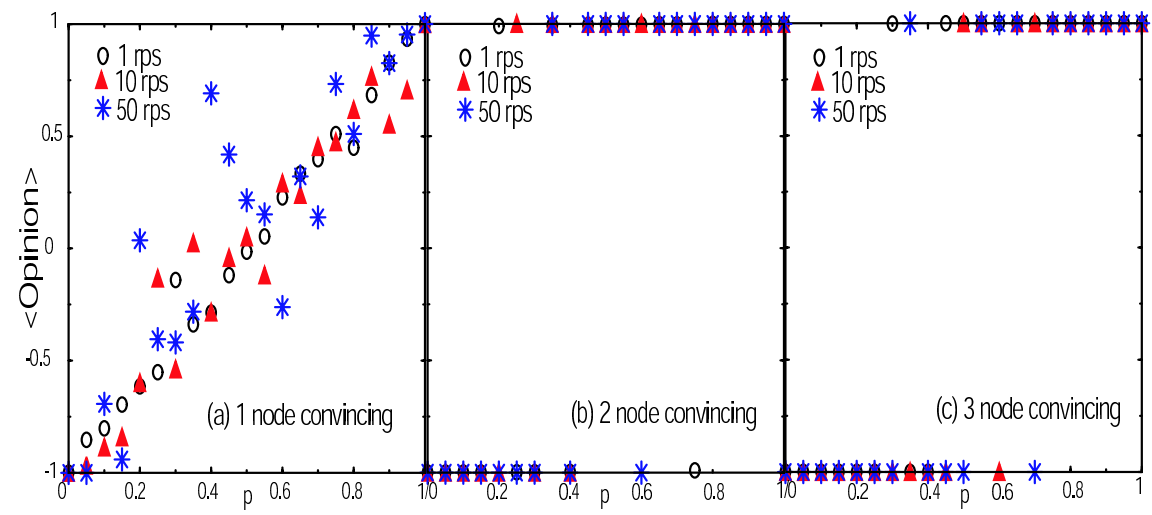

Figure 2: Sznajd model on a 29576 nodes pseudo-fractal network with $N_{s}=1$, 10 and 50 runs per time step for (a) 1 node convincing, (b) 2 nodes convincing and (c) 3 nodes convincing.

- 3 sites convincing: For each $i$ site chosen, we select 2 of its neighbors at random. If all these three sites have the same opinion, they change the opinion of all their neighbors.

Figure (2) shows the mean opinion for the three rules (1, 2 and 3 sites convincing) for different initial concentrations $p$ and $N_{s}$ Sznajd runs per step (rps): $1 \mathrm{rps}\left(N_{s}=1\right)$ corresponds to one realization of Sznajd per step, that is at each step, one chooses $3^{t}$ nodes of the network at random to simulate Sznajd. $10 \mathrm{rps}\left(N_{s}=10\right)$ and $50 \mathrm{rps}\left(N_{s}=50\right)$ correspond, respectively, to choose $10 \times 3^{t}$ and $50 \times 3^{t}$ nodes randomly per step. We let the network grow up to 29576 sites, that corresponds to 10 steps. For 1 node convincing, the model can not reach consensus, the results become more unpredictable, the larger the number of runs per step (Fig 2 ). However, for two and three nodes convincing, a full consensus is observed (Figs 20 and 2 :). We clearly see that for rules 2 and 3 one has a 1 st order transition since the order parameter (opinion) jumps drastically and shows strong hysteresis. Our results are very similar to the ones obtained on the Barabási-Albert network when the same rules are applied [15], except that the latter requires more Sznajd runs for the network to reach a fixed point, i.e., a full consensus.

Since after some time steps the rules 2 and 3 always lead to a consensus and the whole system reaches a fixed point, in Fig 3 we show the number of samples, out of 1000, for which the fixed point all "up" (all with opinion +1 ) is obtained when different values for the initial concentration $p$ of nodes "up" are simulated. As we can see, the fraction of realizations with fixed point "up" depends on the probability $p$ and on the implemented rule. This is opposed to the results obtained with the same rules (2 or more nodes convincing) on a square lattice, where an abrupt change is observed for $p \geq 0.5$ [19; but in agreement with the ones on a Barabási-Abert network [15] and on a 1-dimensional chain [16]. For 


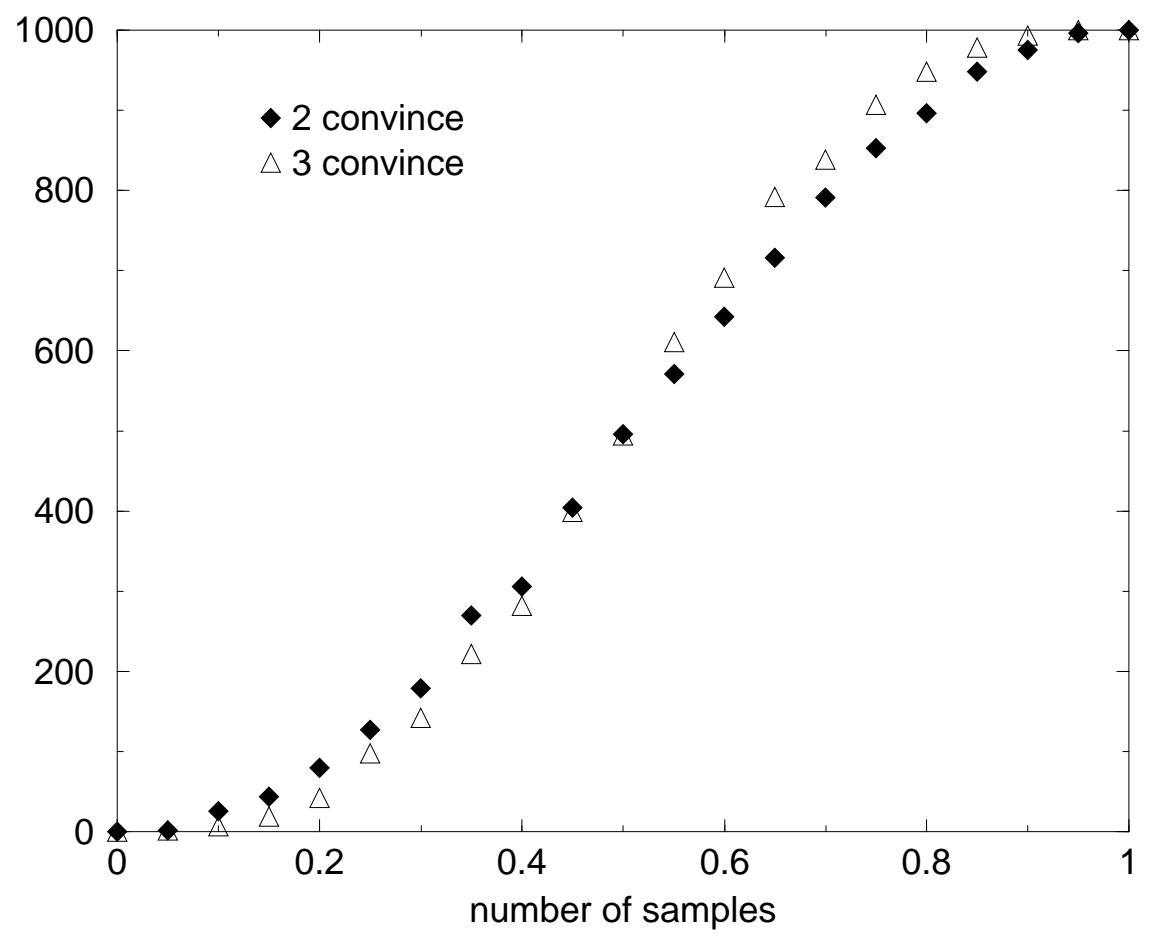

Figure 3: Monte Carlo simulation on a 29576 nodes pseudo-fractal network counting the number of samples, out of 1000 , for which the fixed point all "up" is obtained when different values for the initial concentration $p$ of nodes "up" are simulated. As we can see, the fraction of realizations with fixed point up depends on the probability $p$ and on the rule implemented, for Sznajd models with 2 and 3 nodes convincing.

1 node convincing the system does not tend to a fixed point (consensus), while on square lattices it does [17, 18, although without the abrupt change of 2 sites convincing.

\subsection{Simulation of elections with many candidates}

We create a network of interacting nodes by using the pseudo-fractal network prescription as described before (Eq11). In addition to our network, we also simulated the rule presented in 12 for modeling elections on a Barabási network. We summarize the generation of the Barabási network as follows: At the beginning of the simulation we have 6 fully connected nodes. After that, we add more and more nodes and connect it with 5 of the present nodes chosen at random. Thus the growth probability at any existing node is proportional to the number of nodes already connected to it. For comparison we generate 


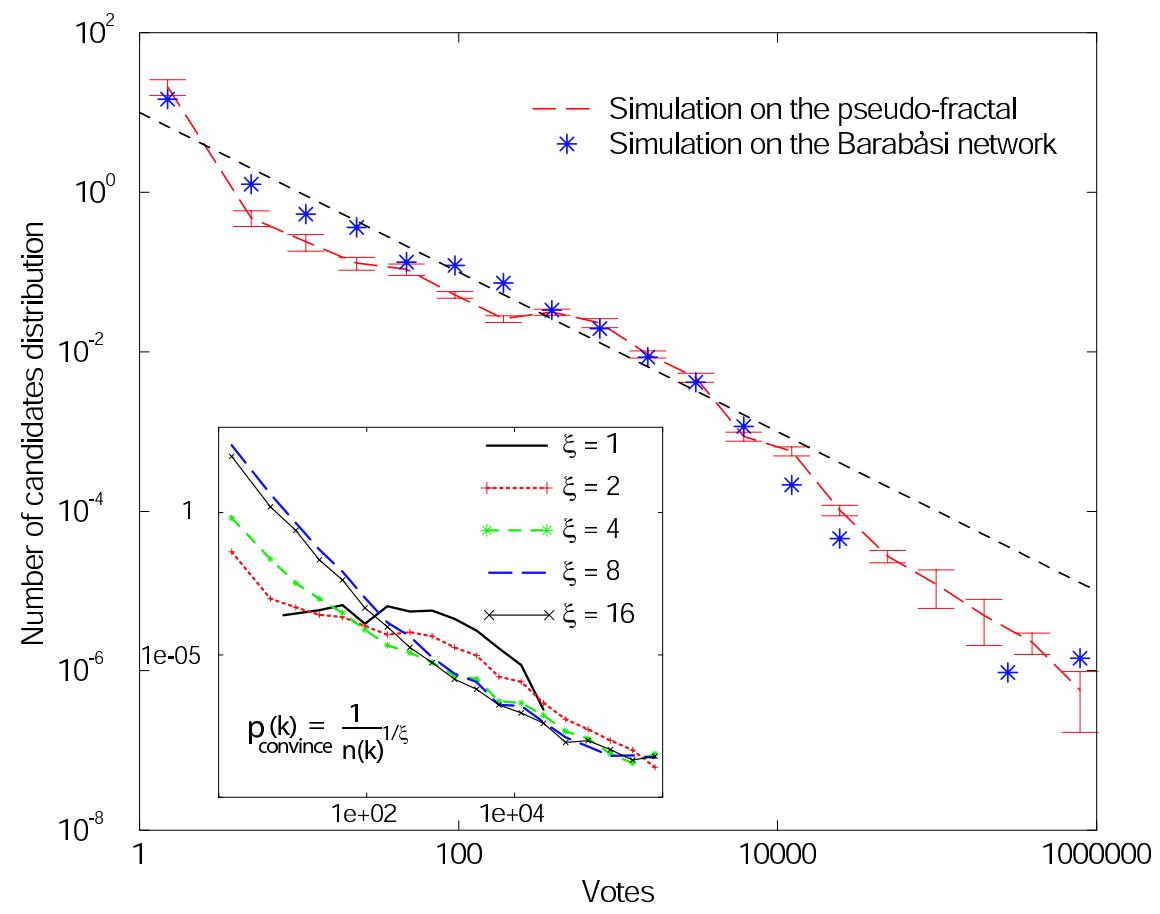

Figure 4: Plot of the voting distribution of an election process on the pseudofractal compared to the simulation on a Barabási network. Both networks have 797163 sites (voters) and 500 candidates. We average over 20 realizations for each network. The distribution for the pseudo-fractal is after 15 iterations of the convincing process with $\xi=2$ (Eq. 3) . The distribution for the Barabási network is after 83 iterations. In the inset we show the results of the simulation on the pseudo-fractal for $\xi=1,2,4,8$ and 16 , after 400, 20, 4, 2 and 1 iterations respectively. We see that the results depend on the rule chosen.

both networks with the same number of nodes (voters), and the same number of candidates.

After generating a network, one starts with the "election process". $N$ candidates are randomly distributed. The value $n(1 \leq n \leq N)$ of a node on the network represents that this voter has preference for the candidate $n$. Initially, all sites start with value zero, meaning that there is no preference for any candidate, except for $N$ sites that have the number of a candidate. Now, the electoral campaign starts (only voters with preference for a candidate can influence other voters, à la Sznajd). At each time step all the nodes are randomly visited: a random list of nodes assures that each node is reached exactly once. For each visit, we implement the following process:

- We choose a node $i$ at random, if it has preference for a candidate, we choose among its connected nodes, a node $j$ at random. Otherwise, we 


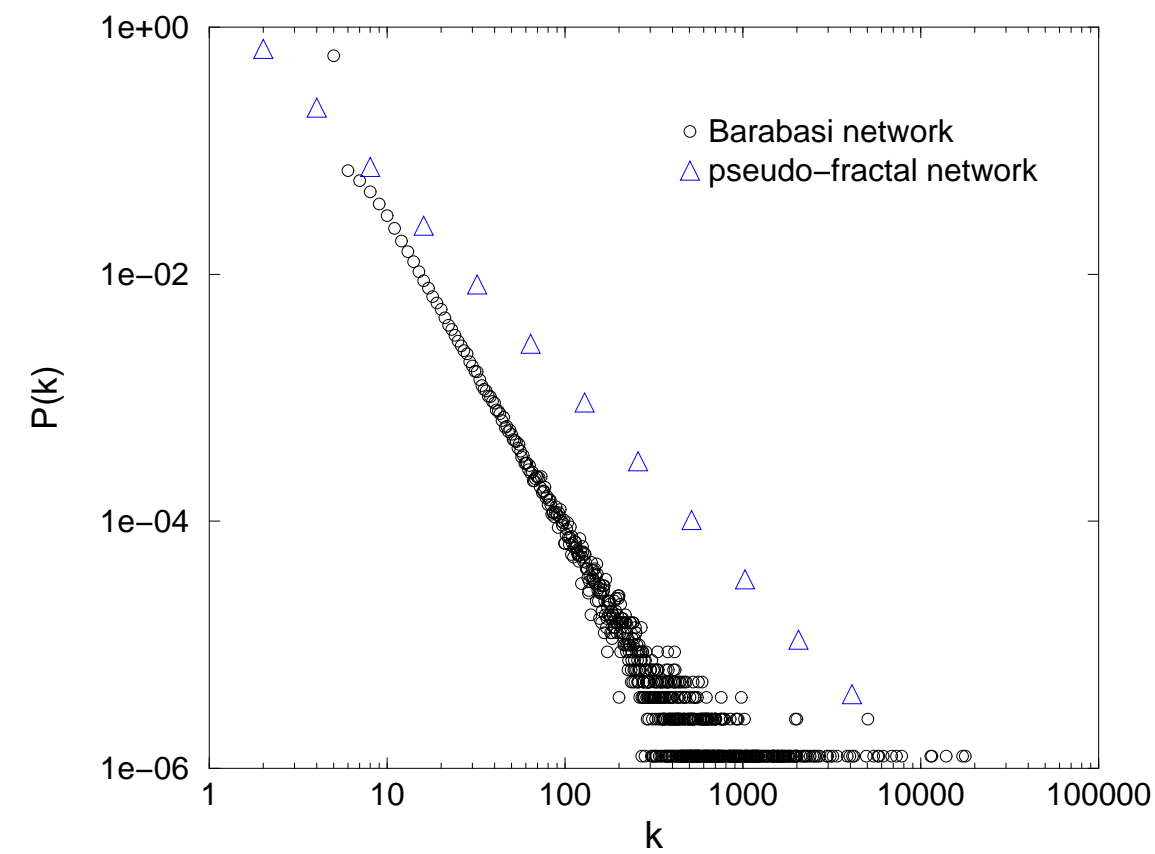

Figure 5: Comparison of the degree distributions of the Barabási network $P(k) \sim k^{-\gamma}(\gamma=2.9)$, and of the pseudo-fractal network, $P(k) \sim k^{1-\gamma}$ $(\gamma=1+\ln 3 / \ln 2)$.

randomly select another node.

- If node $j$ has the same candidate as node $i$, each node convinces all the nodes connected to it with probability:

$$
p(k)=\frac{1}{n(k)^{1 / \xi}}
$$

where $n(k)$ is the number of nodes connected to either $i$ or $j$, and $\xi>1$ a switching factor that is analyzed later.

- If node $j$ has no candidate, node $i$ convinces it to accept its own candidate with the probability $p(i)$ of eq. (3).

- If node $j$ has a different candidate from node $i$, we choose another node $i$.

As in real elections, we do not wait for a kind of fixed point which here would correspond to all the nodes preferring the same candidate, but we count the votes at an intermediate time. We group in a histogram the number of candidates which received a certain number of votes. Because the bin size for the votes increases by a factor 2 for each consecutive bin we divide each point of 
the histogram by the bin size, for this reason we have numbers lower than one for the histogram of the number of candidates, this kind of "voting distribution" is used in the literature for analyzing similar results [12, 13]. In Fig 4 we see that the results of the voting distribution for the simulations on the pseudofractal and on the Barabási network agree very well, using $\xi=2$ (Eq. 31) for the pseudo-fractal. In the inset we show the results of the simulation on the pseudo-fractal for $\xi=1,2,4,8$ and 16, after 400, 20, 4, 2 and 1 iterations, respectively. The results differ for each selection of $\xi$ : the system reaches a fixed point more rapidly for larger parameter $\xi$, i.e, one needs less iterations in order to reach the same distribution's width. Using the same value used in the simulations on the Barabási network, $\xi=1$, after nearly 2000 iterations, a fixed point (consensus) on the pseudo-fractal network could not be reached. A

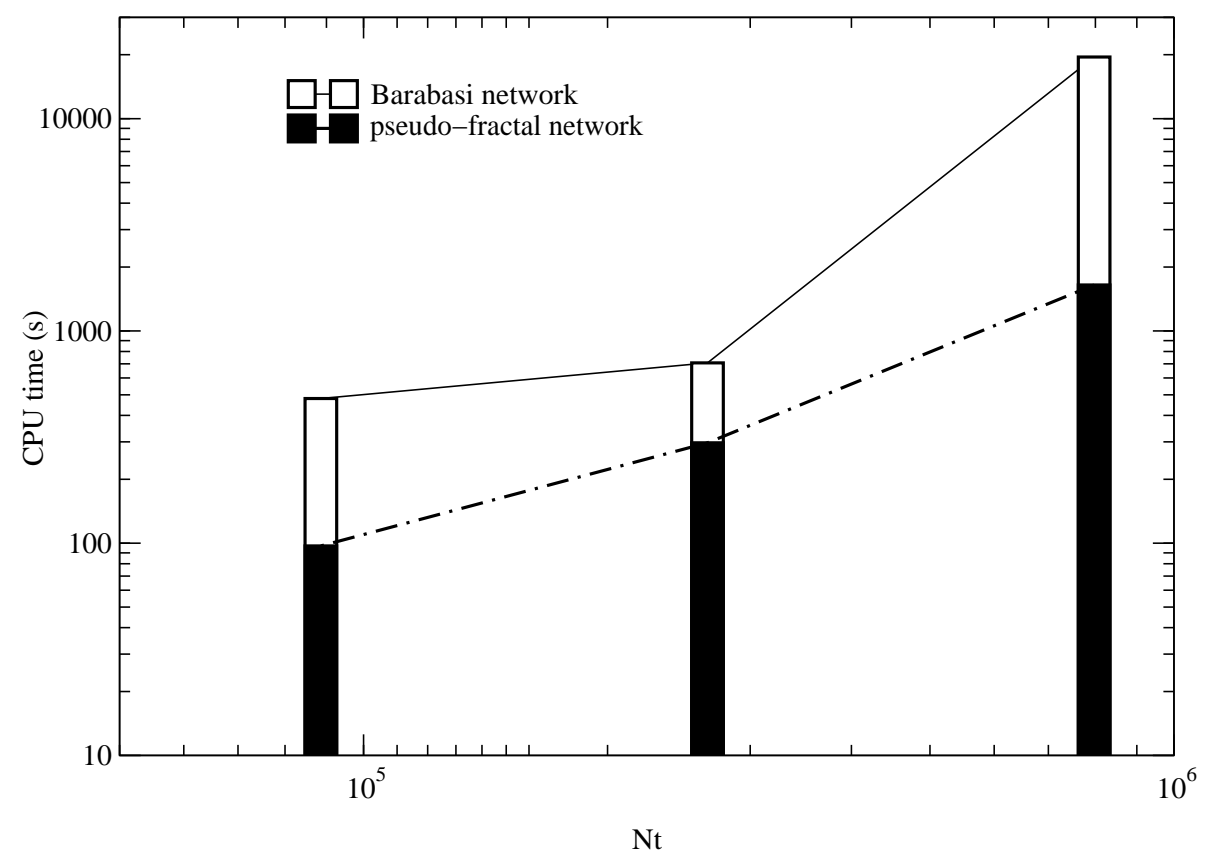

Figure 6: CPU time (seconds) on a PIV 2.4 GHz vs. size of the network for the pseudo-fractal network and for the Barabási network. We simulate an election process with 100 iterations of the convincing model, on networks of sites: 88575, 265722 and 797163 with 500 candidates. The computation time on the pseudofractal grows linearly with the size of the system, while for the stochastic network it grows exponentially.

For the simulation on the Barabási network we use $\xi=1$, like reported in [12]; this selection means that on average each node convinces one other node at 


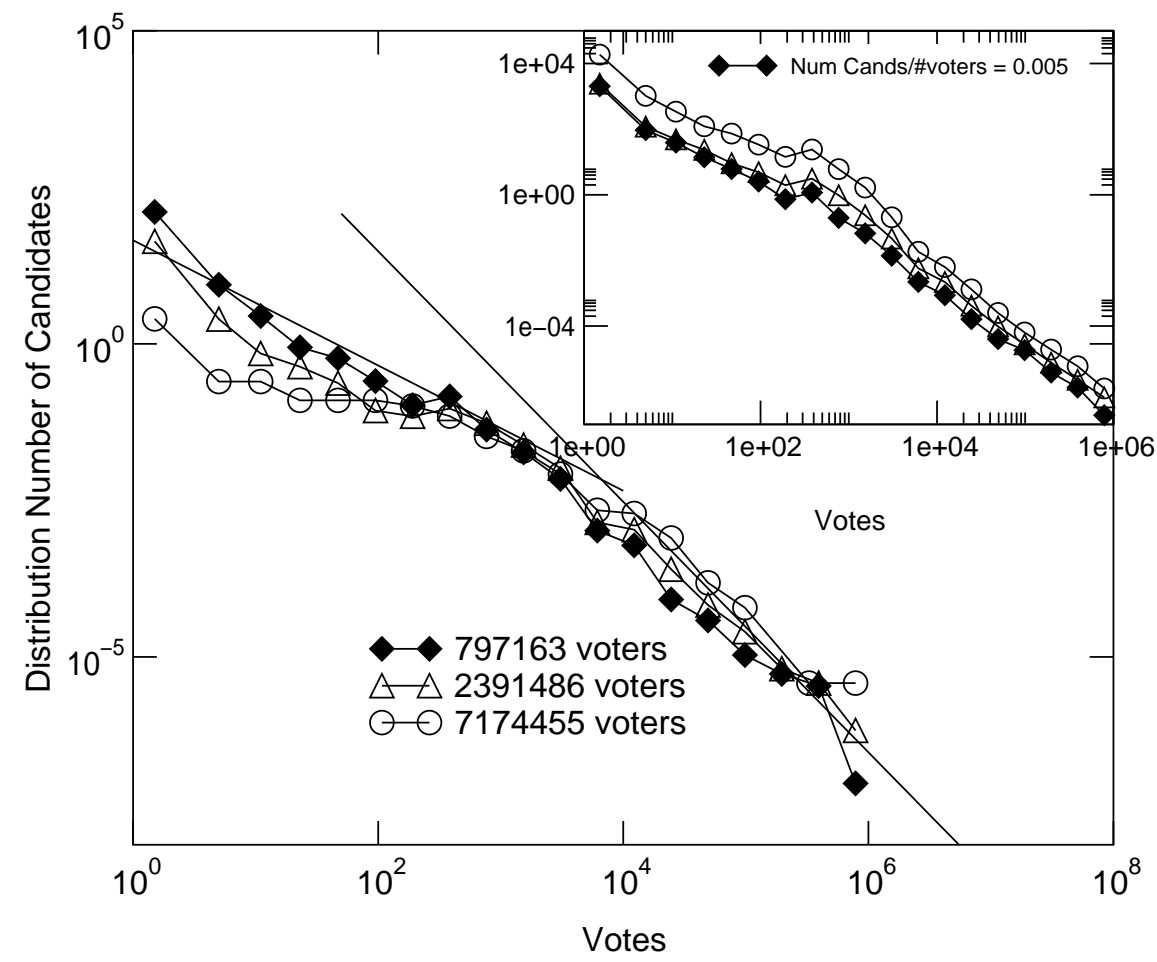

Figure 7: Test for size effects on the distribution of candidates for the simulation of elections on the pseudo-fractal with 12 generations (diamonds), 13 generations (triangles) and 14 generations (circles) with 2000 candidates each, after 15 iterations of the election process. In the inset we make the simulation with a number of candidates proportional to the number of sites of the network, keeping the relation \#Cands/\#voters $=0.005$, we see that the results are size-independent. The solid lines are guides to the eye with slopes -1 and -2 .

each process. As we show in Fig. 5 the degree distribution of the pseudo-fractal is discrete and is given by $P_{\text {cum }}(k)=k^{-\ln 3 / \ln 2}$, while the degree exponent for the Barabási network presented in this work is $\gamma=2.9$ [11. Knowing $P(k)$ we can calculate the probability distribution of convincing choosing one site at random, that is the probability of convincing Eq3 multiplied by the degree distribution $P(k)$. For the convincing distribution of both networks to be similar, one has to chose Eq. 3 with $\xi=2$ for the simulation on the pseudofractal. We obtain qualitatively the same results in both simulations, being 12 time faster in the pseudo-fractal than in the Barabási network, because it requires less memory space and computation time (see Fig [6).

In Fig. 7 we see that the shape of the voting distribution after a given number of iterations does not change with the size of the network. Changing 


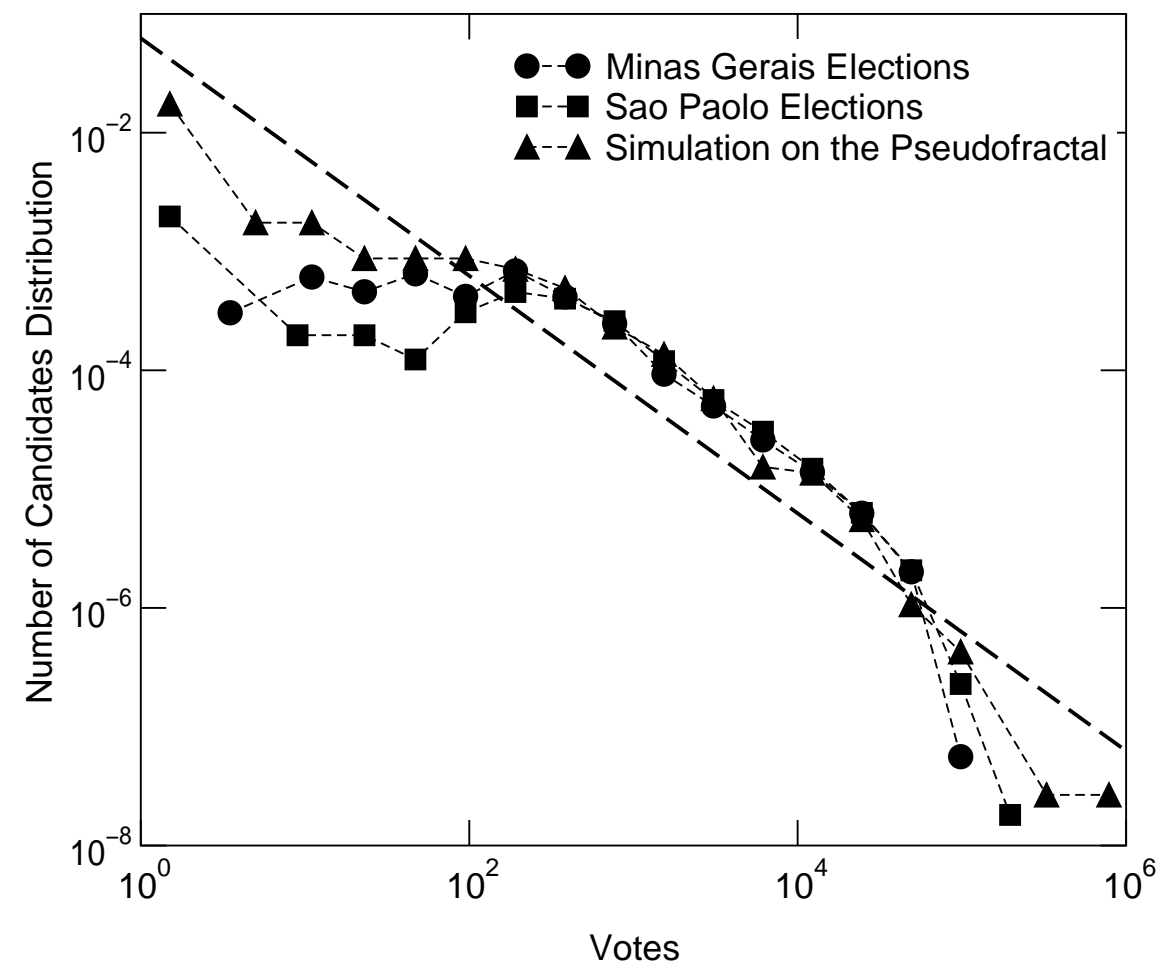

Figure 8: Result of the simulation of an election process after 20 iterations on a pseudo-fractal network of 21523362 nodes and 1144 candidates (triangles). Compared to the voting distribution for the state of Minas Gerais in 1998 (circles) (819 candidates, 11815183 voters) and the state of São Paulo (1998) (squares) (1260 candidates, 23321034 voters). Both axis are plotted on a logarithmic scale. The dashed straight line is a guide to the eye with slope -1 . The bin size for the votes increases by a factor 2 for each consecutive bin. The height of the distribution of the pseudo-fractal is multiplied by 10 to see better the comparison of the results.

We consider the results from two Brazilian states (São Paulo and Minas Gerais) for the positions of local state deputies. In such elections the voters vote directly for the candidate and not for parties. Some elections occur with a high number of candidates, of the order of thousands, and with a number of voters of the order of millions. The official results for each state are available on the Internet [20].

In Fig 8 we see the results of a simulation on a 15-generations pseudo-fractal (21523362 nodes) and 1144 candidates, and compare it to the results of real elections described in Fig 8 . For the moment we do not take into account ab- 
stentions, or invalid votes in the simulation. The pseudo-fractal tends to consensus in a similar way as the real elections. After averaging over more than 100 realizations we see, that the deviations from a perfect line in the intermediate regions of votes, seen in the real elections, are not of statistical nature but seem due to the determinism of the network. The general behavior of the distribution of candidates of the simulation of the elections on the pseudo-fractal however agrees with the one observed in real results. The results of the distribution of candidates for the simulated and the real cases follow hyperbolic law,

$$
N(v) \propto 1 / v,
$$

for the number $N$ of candidates having $v$ votes, extending over two or three order of magnitude, with deviations only for small and large numbers of votes [13.

In addition we analyzed the behavior of the voting distribution for the elections in India to the lower house of the Parliament (Lok Sabha). These elections are events involving political mobility and organizational mobility on an amazing scale. In the 1998 election to Lok Sabha there were 1269 candidates from 38 officially recognized national and state parties seeking election,1048 candidates from registered parties not recognized and 10635 independent candidates. A total number of 596185335 people voted. The Election Commission employed almost 400000 people to run the election. The official results of this elections are available on the Internet 21. In contrast to the Brazilian elections, in this process the country is divided into 543 parliamentary constituencies, each of which return one representative to the Lok Sabha. That is, not all the voters elect among all the candidates of one state (like in Brazil), but there is one election process for each of the 543 representatives, that occurs in each parliamentary constituency. These parliamentary constituencies are selected by an independent Delimitation Commission, which creates constituencies which have roughly the same population, subject to geographical considerations and the boundary of the states and administrative areas.

At the end of the process one can analyze the voting distribution of each state of the India. In Fig. 9 we observe the results for 5 states: Uttar Pradesh, Goa, Andhra Pradesh, Haryana and Kerala, with 85, 2, 42, 10 and 20 constituencies respectively. The voting distribution for each state is the superposition of different electoral processes for all the constituencies of the state. For each election in a constituency there is a voting result that corresponds to few candidates, between 5 and 10. The difference between the India and Brazilian results appears at this stage. If one analyses the election process of each parliamentary constituency, even though one does not have a large number of candidates for the statistics, a distribution with slope $\sim-1$ is observed. However, the final results of the process for each Indian state provide a different profile far from the hyperbolic one (1/v type of distribution) (see Fig 9), which was observed for many Brazilian states [12, 13]. In order to analyze the number of candidates $N$ which received a certain fraction of votes $v$ for the nationwide voting process, we have normalized the votes of each candidate by the total number 


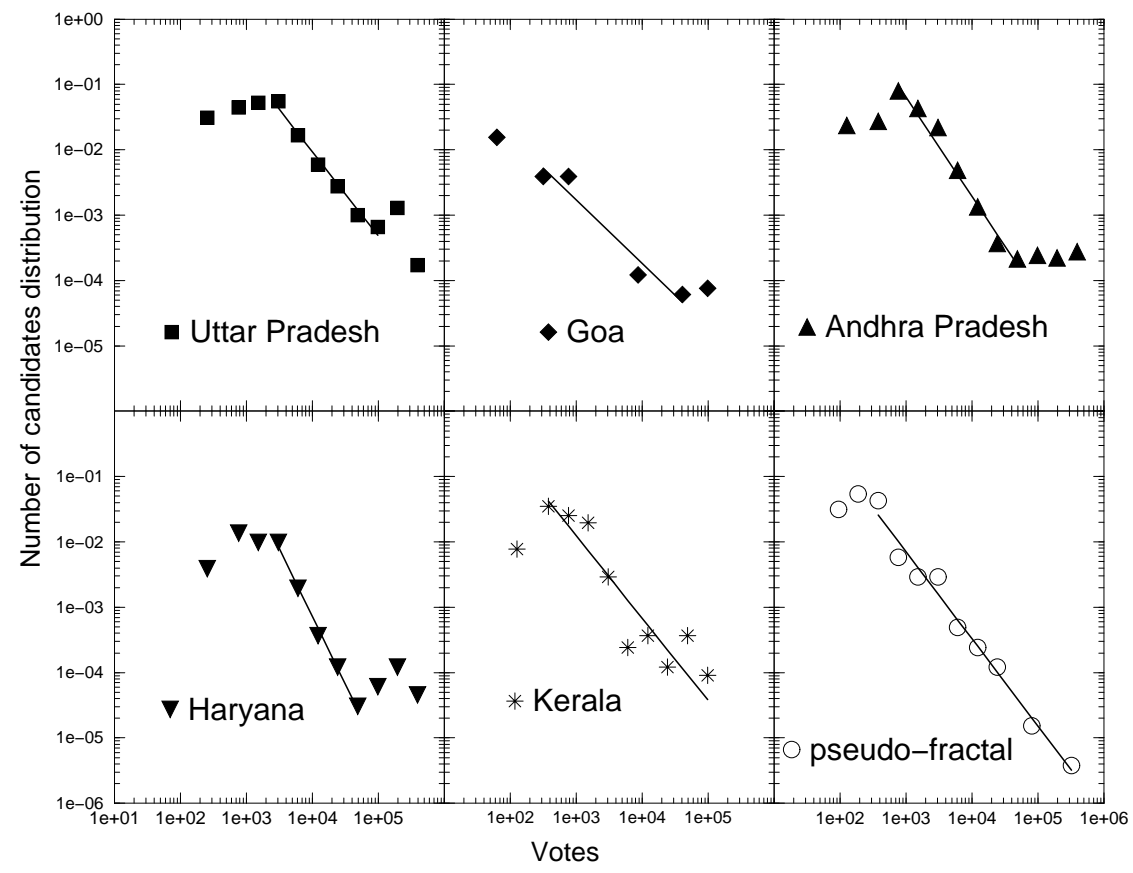

Figure 9: Voting distribution for state elections of India in 1998. For Uttar Pradesh (squares) with 55, 015, 804 voters and 649 candidates. Goa (diamonds) with 532,766 voters and 12 candidates. Andhra Pradesh (triangles up) with $31,829,338$ voters and 301 candidates. Haryana (triangles down) with 7516, 884 votes and 84 candidates and Kerala (stars) with 13,036,581 voters and 108 candidates. We obtain results qualitatively comparable to this kind of processes with a Pseudo-fractal with 12 generations and 80 candidates, after few iterations (5). The solid lines are squares fits to the data, in the intermediate regions. The slopes are: -1.32 (Uttar Pradesh), -0.97 (Goa), -1.51 (Andhra Pradesh), -2.06 (Haryana), -1.26 (Kerala) and -1.32 (Pseudo-fractal)

of voters (Fig 101). As can be seen, the number of candidates $\mathrm{N}$ follows a power law $N(v) \propto v^{\alpha}$, with $\alpha=-1.3$ (for Brazil, $\alpha=-1$ ).

The differences in the Sznajd simulations on the pseudo-fractal network for the India and Brazilian elections are mainly due to the number of candidates considered for each one. In the latter, the number of candidates is almost $0.005 \%$ of the lattice nodes, while for the former it is $0.01 \%$. The fixed point (consensus) is reached the faster, the larger the density of candidates. Because of that, the comparison of the real elections in India with our simulations were made taking account only 5 iterations of the Sznajd model on the pseudo-fractal network (20 iterations for Brazilian elections). 


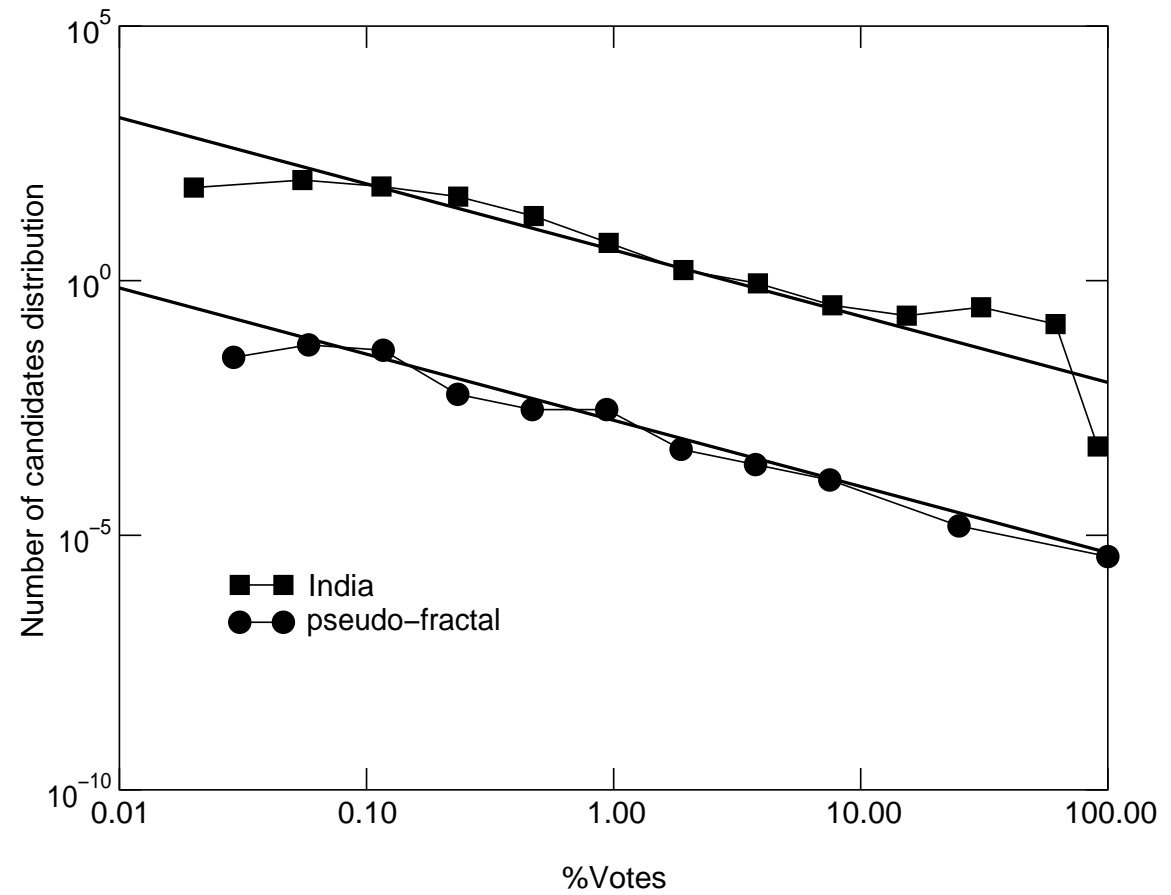

Figure 10: Voting distribution for India in 1998 (squares), compared to the simulation on a Pseudo-fractal (circles) with 12 generations and 80 candidates, after few iterations (5). The solid lines are guide to the eye with slope -1.3

\section{Conclusions}

We studied the behavior of a deterministic scale-free network simulating a spreading of opinion model on it. We solved the Sznajd's model on a growing deterministic scale-free network, obtaining always a consensus of opinion after some runs of the model. The final opinion presents a 1 st order transition, that shows strong hysteresis, as a function of the initial concentration of opinions in the network for rules 2 and 3 . The results coincide with the results reported for a stochastic scale free network [15]. We found that there is not scaling in the finite-size cut-off showing that the system is not critical (Fig [7). We simulated election processes on the network. The probability of convincing of the model has to be adapted $(\xi=2$, in Eq. 3) in order to avoid the differences in the values of the exponents $\gamma$ of the degree distribution of our deterministic network and the $\gamma$ exponent of a typical stochastic network (Fig. 44. We obtained the same results, reported for a stochastic scale-free network, but with computation times considerably lower (see Fig 6). We could use our model to reproduce qualitatively good complex electoral processes, such as the states elections for parliament in Brazil and India. 


\section{Acknowledgments}

We thank D. Stauffer for helpful discussions and a critical reading of the manuscript; A.O. Sousa acknowledges a research grant from the Alexander von Humboldt Foundation and M.C. González a fellowship from DAAD.

\section{References}

[1] S.H. Strogatz et. al., Nature 410, 268 (2001).

[2] R. Albert and A.-L. Barabási Rev. Mod. Phys. 74, 47 (2002).

[3] A.-L. Barabási, E. Ravasz and T. Vicsek Physica A 299, 3 (2001).

[4] S.N. Dorogovtsev, A.V. Goltsev and J.F.F. Mendes, Phys.Rev. E 65, 066122 (2002).

[5] S.N. Dorogovtsev and J.F.F. Mendes, Evolution of Networks: From Biological Nets to the Internet and $W W W$, Oxford University Press, Oxford (2003).

[6] M.E.J. Newman, SIAM Review 45167 (2003).

[7] D. Stauffer, Journal of Artificial Societies and Social Simulations 5, issue 1, (jass.soc.surrey.ac.uk) (2002); D. Stauffer, Conference 50th Anniversary of the Metropolis Algorithm (in press) (2003) = cond-mat $/ 0307133$

[8] S. Galam, Y. Gefen and Y. Shapir, Math. J. of Sociology 9, 1 (1982); S. Galam, J. of Math. Psychology 30, 426 (1986); S. Galam and S. Moscovici, Euro. J. of Social Psy. 21, 49 (1991); S. Galam, Eur. Phys. J. B 25 Rapid Note, 403 (2002); S. Galam, Physica A 320, 571 (2003).

[9] K. Sznajd-Weron and J. Sznajd, Int. J. Mod. Phys. C 11, 1157 (2000).

[10] G. Defuant, D. Neau, F. Amblard, G. Weisbuch, Adv. Complex Syst. 3, 87 (2000); G. Weisbuch, G. Defuant, F. Amblard, J.-P. Nadal, Complexity 7, 55 (2002); G. Defuant, F. Amblard, G. Weisbuch, T. Faure, J. Artificial Soc. Social Simulation 5, issue 4, paper 1 (2002); R. Hegselmann, M. Krause, J. Artificial Soc. Social Simulation 5, issue 3, paper 2 (2002); U. Krause, p.37 Modellierung und Simulation von Dynamiken mit vielen interagierenden Akteuren, edited by U. Krause and M. Stöckler, Bremen University (1997).

[11] R. Albert and A.-L. Barabási, Science 286, 509 (1999).

[12] A.T. Bernardes, D. Stauffer, and J. Kertész, Eur. Phys. J. B 25123 (2002).

[13] R.N. Costa Filho, M.P. Almeida, J.S. Andrade, Jr., and J.E. Moreira, Phys. Rev. E., 60, 1067, (1999); M.L. Lyra, U.M.S. Costa, R.N. Costa, J.S. Andrade, Europhys. Lett., 62, 131 (2003); R.N. Costa, J.E. Moreira, J.S. Andrade, Physica A, 322, 698 (2003). 
[14] K. Sznajd-Weron and J. Sznajd,Int. J. Mod. Phys. C 13, 115 (2002).

[15] J. Bonnekoh, cond-mat/0305125.

[16] K. Sznajd-Weron and J. Sznajd, Int. J. Mod. Phys. C 11, 1157 (2000).

[17] C. Schulze, Int. J. Mod. Phys. C 14, 95 (2003).

[18] R. Ochrombel, Int.J. Mod. Phys. C 13, 1091 (2001).

[19] D. Stauffer, A.O. Sousa, and S. Moss de Oliveira, Int. J. Mod. Phys. C 11, 1239 (2000).

[20] http://www.tse.gov.br/ele/

[21] http://www.indian-elections.com/states/ 